\title{
Experten und Computerspieler über eine Art, in die virtuelle Realität einzutauchen
}

\author{
Beata Medyńska-Gulij*, Krzysztof Zagata \\ Adam Mickiewicz University in Poznań, Poland, Department of Cartography and Geomatics, Beata Medyńska-Gulij- \\ bmg@amu.edu.pl, Krzysztof Zagata - krzysztof.zagata@amu.edu.pl \\ * Corresponding author
}

Keywords: Evaluation of cartographic multimedia; expert opinion; gamer opinion; immersion way; graphical enrichment; medieval stronghold; virtual reality; cartographic materials; historico-geographical space; cultural heritage.

\begin{abstract}
:
In dieser Studie haben wir das Problem der Bewertung der Methode des Eintauchens (Immersion) in einen speziellen historisch-geographischen Raum, der auf der Grundlage traditioneller kartographischer und graphischer Materialien konstruiert wurde, behandelt. Als Basis dienten die Meinungen von zwei Nutzergruppen hinsichtlich der Wahrnehmung von Kulturobjekten, die in einer den Nutzern bisher unbekannten virtuellen Realität rekonstruiert wurden. Die Untersuchung war in vier Forschungsphasen gegliedert: (1) Ermittlung von Konzepten verschiedener Forscher durch Erörterung zweier Arten von Ansätzen, (2) Erarbeitung einer VR-Anwendung gemäß dem Schema, das auf Wissen aus analogen Quellen und digitalen Aktionen in mehreren Arbeitsbereichen basiert, (3) Vorbereitung und Durchführung einer Umfrage unter Experten und Spielern, (4) graphische Gegenüberstellung der Ergebnisse der Umfrage und Formulierung von Schlussfolgerungen. Die Bewertung der spezifischen Art des Eintauchens in die VR der aktuell rekonstruierten Gebäude durch Experten für mittelalterliche Burgen und erfahrene Nutzer von Bildschirmspielen ergab einen erweiterten Überblick über die Wirksamkeit und Attraktivität der Immersion sowie Anregungen für weitere VR-Entwurfsprozesse.
\end{abstract}

